\title{
Philosophiques
}

Normand Lacharité, Introduction à la méthodologie de la pensée écrite, Montréal, Presses de l'Université du Québec, 1987, XVI, 235 p.

\section{Vance Mendenhall}

Volume 15, numéro 2, automne 1988

URI : https://id.erudit.org/iderudit/027057ar

DOI : https://doi.org/10.7202/027057ar

Aller au sommaire du numéro

Éditeur(s)

Société de philosophie du Québec

ISSN

0316-2923 (imprimé)

1492-1391 (numérique)

Découvrir la revue

Citer cet article

Mendenhall, V. (1988). Normand Lacharité, Introduction à la méthodologie de la pensée écrite, Montréal, Presses de l'Université du Québec, 1987, XVI, 235 p.

Philosophiques, 15(2), 465-482. https://doi.org/10.7202/027057ar d'utilisation que vous pouvez consulter en ligne.

https://apropos.erudit.org/fr/usagers/politique-dutilisation/ 


\section{ÉTUDE CRITIQUE}

NORMAND LACHARITÉ, Introduction à la méthodologie de la pensée écrite, Montréal, Presses de l'Université du Québec, 1987, XVI, 235 p.

par Vance Mendenhall

«La connaissance des règles suffit à l'arbitre mais non aux joueurs »

R. Blanché

1. Aujourd'hui, une "Introduction à la méthodologie de la pensée écrite » sera inéluctablement prise dans un contexte défini par le sentiment largement répandu d'insatisfaction à l'égard des institutions scolaires. Ce sentiment se fonde sur une évidence: la fréquentation des institutions scolaires, même sur des périodes très longues, n'assure pas aux étudiants l'acquisition des savoirs et savoir-faire fondamentaux - l'écriture et la lecture - qui permettent d'accéder aux autres savoirs et savoir-faire. La nonacquisition de compétences minimales - savoir déchiffrer un texte et comprendre ce qu'on lit ; savoir composer un texte, intelligible pour autrui rend difficile sinon impossible la réalisation des fonctions de l'école, que celles-ci soient de reproduire la société ou de la contester, de démocratiser l'enseignement ou de libérer les individus. Quoi qu'il en soit, la nonacquisition de compétences minimales produit un effet d'atrophie sinon de privation ; elle engendre des problèmes pour les individus, pour les institutions, pour la société.

1.1 Dans ce contexte, une «Introduction à la méthodologie de la pensée écrite " sera mise à contribution pour résoudre le problème de l'acquisition des compétences minimales, des savoirs et savoir-faire fondamentaux - la lecture et l'écriture. L'utilisateur y cherchera les instruments et les dispositifs qui favorisent l'acquisition d'une maîtrise pratique des règles et des principes définitoires des activités de l'écriture et de la lecture. Il y cherchera des consignes, des techniques, des stratégies qui rendent cette pratique facile, commode et efficace.

1.2 Le problème de l'incompétence, dans ce contexte, est central. L'accueil fait à une «Introduction à la méthodologie » sera lié à sa contribution 
à la résolution de ce problème, à sa capacité de rendre ceux qui y ont recours, compétents ou plus compétents.

1.3 Une façon de rendre compte du livre de Normand Lacharité sera de le situer par rapport à ce problème et de demander s'il contribue à sa résolution. Pour ce faire, il faut commencer par mieux identifier le problème qui engendre tant d'insatisfaction à l'égard des institutions scolaires. Il faut essayer de dire ce qui ne va pas, ce que l'étudiant n'est pas en mesure de faire.

\section{II}

2. L'ignorance de la langue est à l'origine des maux qui affligent l'enseignement. Bien sûr, l'emprise médiatique, la complexité et l'atomisation des savoirs, la défaite d'une certaine «culture universelle », la multiplication des foyers d'apprentissage et de socialisation n'y sont point étrangères. Mais l'évidence avec laquelle ces phénomènes explicatifs s'imposent ne doit pas oblitérer le fait que la transmission et la formation des savoirs et des savoirfaire qui font l'objet d'un enseignement passent nécessairement par la langue. Quels que soient les savoirs et savoir-faire qui font l'objet d'un enseignement, ils sont totalement inaccessibles aux personnes qui ignorent la langue.

2.1 Cette ignorance n'est pas seulement une ignorance de la grammaire (morphologie et syntaxe) et du lexique, elle est aussi et peut-être avant tout une ignorance des choses que nous faisons ou que nous pouvons faire dans la langue (parlée ou écrite). En effet, si la langue est à la fois un instrument et une forme de vie sociale, c'est-à-dire si elle est un moyen de faire des choses comme donner des renseignements, poser des questions, expliquer ou justifier une thèse, faire des promesses, nuancer un propos, etc. et une manière pour un locuteur d'agir ou de se comporter à l'égard d'un interlocuteur (réel ou possible), l'ignorance de la langue se manifestera comme l'incapacité de faire ces choses ou de réaliser certaines formes de comportement interindividuel.

2.1.1 Certes l'ignorance du code et l'incorrection syntactico-sémantique sont des empêchements à l'interaction langagière, mais elles ne rendent pas toujours celle-ci impossible. Des locutions comme :

1. «Montage au $2^{e}$ plancher " (affiché près d'un ascenseur dans un hôpital à Ottawa);

2. «Je ne monterai pas deux planchers à pied. »; (Étudiante à l'Université d'Ottawa, 1986);

3. «Prends soin.";

peuvent produire, sans penser à M. d'Iberville-Fortier, toutes sortes d'effets. Mais, dès que nous savons (ou pensons savoir) ce que le locuteur fait (ou essaie de faire), l'interaction langagière est réussie (en partie, du moins).

Inversement, la maîtrise du code et la correction syntactico-sémantique n'assurent pas la réussite de l'interaction langagière. Sans une connaissance 
de la langue comme moyen de faire des choses, ou, plus exactement, comme pratique sociale, nous pouvons nous trouver exclus de l'interaction réalisée par des locutions telles:

4. Savez-vous qui est le premier ministre du Canada?

5. Le déclin de l'été américain.

où (4) peut être une interrogation, ou une information et (5) une information ou un jeu de mots. Ainsi, autant il est important de savoir comment marche la langue et de maîtriser les règles du fonctionnement interne du code, autant il est essentiel de savoir à quoi sert ce code et comment s'en servir.

2.2 L'interlocution est une interaction qui se réalise dès que l'interlocuteur reconnaît ce que le locuteur fait ou essaie de faire, et qui échoue lorsque l'interlocuteur ne reconnaît pas ou ne peut pas reconnaître ce que le locuteur fait ou essaie de faire.

L'interlocuteur peut ne pas reconnaître ce que le locuteur fait ou essaie de faire parce que :

1. Le locuteur ne sait pas faire;

2. L'interlocuteur ne sait pas reconnaître (tel acte)

$\grave{A}$ partir du moment où un locuteur ne peut pas compter sur la reconnaissance (possible) de ce qu'il fait (ou essaie de faire), il ne peut pas le faire: un «jeu de mots» qui est pris pour une "assertion» ou un " renseignement » n'est pas un « jeu de mots », une argumentation prise pour une suite d'informations ne peut pas produire les effets d'une argumentation.

2.3 L'incapacité de faire des choses comme expliquer, justifier, commenter, concéder, élaborer, décrire, circonstancier, etc., et de les reconnaître lorsqu'elles sont faites (ou lorsqu'on essaie de les faire) s'est installée dans les institutions scolaires, et ailleurs. S'il faut attendre les résultats d'études empiriques pour mesurer l'amplitude de ce phénomène, il est d'ores et déjà possible de dire qu'il se produit quelque chose comme un rétrécissement ou une contraction dans le répertoire commun des interactions langagières de sorte qu'il ne reste, comme le dit Baudrillard, que deux modes : l'assertorique (c'est ainsi) et l'injonctif (faites). On a l'impression que nous ressemblons de plus en plus à ce menuisier que décrit Georges Perec dans La vie mode d'emploi (Paris, Hachette, 1978, p. 148) qui travaille dans un atelier où se trouvent tous les instruments du métier - trusquin, bouvet, gorget, varlope, bédane, riflard, guillaume, scie à araser, égoïne, etc. - et qui les appelle tous " machin » et, faute de savoir à quoi ils servent, utilise le même " machin » pour refendre, couper, dégrossir, raboter, buriner, etc. Ou encore nous sommes comme un cuisinier qui renonce à l'emploi d'instruments spécialisés et coupe, pèle, gratte, hache, dépèce, pique, etc. avec le même couteau. Comme ce menuisier et ce cuisinier font maladroitement ce qu'ils font et se trouvent dans 
l'impossibilité de réaliser certaines opérations culinaires ou de fabrication, de même, limités à l'assertion et à l'injonction, nous nous trouvons à faire certaines choses avec maladresse et à ne pas pouvoir en faire d'autres, à ne pas pouvoir nous engager dans certaines formes d'interaction.

2.4 Les choses que nous faisons dans le langage, les relations que nous instaurons ou contribuons à instaurer par là, ou les effets que nous produisons, ne sont pas le résultat de l'exploitation de quelque «fonds » naturel. Le fait que l'emploi des mots et des suites de mots - les formes grammaticales, les mots du lexique, les tournures et les constructions, comme le dit Oswald Ducrot - a pour caractéristique régulière d'instaurer entre les interlocuteurs des relations spécifiques n'est pas un fait de nature. Il est un fait historicosocial, c'est-à-dire, une acquisition résultant d'un apprentissage. Nous apprenons qu'en employant tels mots, telles formes grammaticales, nous faisons ou pouvons faire telle chose - nous faisons une promesse ou nous offrons des condoléances ou nous justifions une opinion ou une action, par exemple. Nous apprenons que dans notre groupe socio-linguistique, pour faire une promesse, donner un renseignement, justifier une idée, offrir des condoléances, nous devons employer tels mots, telles formes grammaticales, telles tournures, telles constructions, sous peine de faire autre chose ou rien du tout. De même nous apprenons que par le fait d'offrir des raisons nous pouvons convaincre ou persuader; ou encore qu'en ordonnant nos mots de telle manière nous aménageons un chemin, un parcours qu'un interlocuteur (réel ou possible) suivra (ou pourra suivre). Nous apprenons ce qui compte comme comportement socialement acceptable, nous apprenons à réagir, à recevoir, à interagir ; nous apprenons, par exemple, que le refus d'une argumentation est « déraisonnable », irrationnel, à moins qu'il ne soit lui-même l'objet d'une argumentation.

Ainsi, la production et la réception d'actes langagiers sont des activités apprises et socialement réglées. Pour s'engager dans ces activités, pour faire quelque chose avec les mots, il faut une connaissance pratique de ces règles. L'ignorance ou la méconnaissance de ces pratiques, de ces "façons de faire ", de ces règles, suffit pour perturber l'interaction langagière sinon pour la rendre impossible.

2.5 Le problème de l'École n'est ni celui de mauvaises notes, ni celui de la non-acquisition de «connaissances fondamentales». Le problème est celui de l'incapacité de faire les choses (et celle de les reconnaître lorsqu'elles sont faites) que l'acquisition de ces «connaissances fondamentales» suppose. Le problème est (en partie) celui de ne pas savoir faire des choses comme expliquer, justifier, commenter, élaborer, décrire, etc. et de ne pas savoir les reconnaître lorsqu'elles sont faites.

Autrement dit, le problème de l'École, une partie des difficultés qu'éprouvent les enseignants dans la réalisation de leurs tâches et une partie des déceptions de ces mêmes enseignants, proviennent du fait qu'un trop grand nombre d'érudiants à tous les niveaux - secondaire, collégial, universitaire - n'ont pas une maîtrise adéquate de ces savoir-faire. Ils sont incapables de faire les choses qu'on leur demande : reconnaître les thèmes et les propos, la 
hiérarchisation des thèmes et des propos, reconnaître les rapports entre les éléments d'un discours, les types de discours (narration, description, exposition, commentaire, argumentation, etc.) faire des résumés, problématiser un thème ou un propos, circonstancier un thème ou un propos, évaluer la force et la pertinence des rapports posés entre les éléments du discours, etc.

III

3. Pour résoudre ce problème, il ne suffit pas seulement d'améliorer les cours de langue, d'en augmenter le nombre, d'introduire des cours de rattrapage ou de faire faire des dictées publiques. Les savoir-faire ou les compétences dont il s'agit ne concernent pas la connaissance du code mais son emploi.

Bien entendu, certains savoir-faire langagiers sont liés à des champs spécialisés et n'ont de sens qu'à l'intérieur de ces champs : faire un contrat ou un protocole de recherche, invalider une loi ou un projet de loi, institutionnaliser quelqu'un, sont des actes qui dépendent d'un champ (et d'un pouvoir). Mais certains savoir-faire - affirmer, argumenter, décrire, interroger, s'exclamer, par exemple - sont communs à tous les champs, tout au moins ils sont nécessaires pour l'accomplissement des tâches qui reviennent à l'École. Les enseignants supposent ces savoir-faire.

3.1 Cependant une telle supposition n'est légitime que si les enseignants peuvent supposer en même temps que, quelque part dans la chaîne pédagogique, les étudiants ont appris ou pouvaient apprendre ces savoir-faire. Mais ils savent qu'ils ne peuvent pas(plus) faire cette supposition. Ils savent que ces savoir-faire ne font pas (plus) l'objet d'un enseignement. C'est pourquoi ils chercheront dans un livre de méthodologie des instruments, des techniques et des stratégies qui favoriseront l'acquisition et le développement de ces savoir-faire, d'une véritable compétence langagière.

3.2 Cette compétence sera définie, d'une façon minimale, comme la capacité de s'engager dans une interaction langagière (texte ou conversation), c'est-à-dire, la capacité de produire et d'interpréter des actes langagiers. Elle suppose une connaissance opérationnelle des règles, principes et pratiques qui font en sorte que, par le fait d'employer tels mots, de telle façon, dans telles circonstances, un locuteur produit régulièrement tel effet dès que la position ou le rapport de ces mots, ces constructions, ces circonstances aux règles, principes et pratiques est identifié par un interlocuteur (réel ou possible).

3.3 Autrement dit, plus particulièrement, l'enseignant qui recourt à un livre de méthodologie, qui le conseille à ses étudiants ou qui fait un cours autour de lui, le fera pour que ses étudiants soient en mesure de faire certaines choses : des choses «simples» et «fondamentales », mais que les étudiants (et beaucoup d'autres) ne savent pas faire ou ne savent pas faire sans difficultés. Il cherchera dans un livre de méthodologie de quoi leur donner 
les moyens de savoir identifier dans un discours (texte ou conversation) au moins :

- ce dont il est question (thème) ;

- ce qui en est dit (propos);

- ce qui est fait (directement ou indirectement) (affirmer, nier, proposer, décrire, expliquer, illustrer, renseigner, raconter, résoudre un problème, élaborer, motiver, concéder, circonstancier, etc.);

- l'importance relative (principale-secondaire) des thèmes, des propos et des actes langagiers qui composent le discours

Les enseignants peuvent y chercher en plus les moyens d'assurer une connaissance opérationnelle des règles normatives des différents types de discours (discours descriptif, discours d'exposition, discours argumentatif, discours régulatif, etc.) qui déterminent, en partie, l'acceptabilité d'un discours (texte ou conversation).

Enfin, ils peuvent y chercher, outre des moyens d'assurer une compétence minimale d'interprétation et d'évaluation, des moyens d'assurer une compétence minimale de production - non pas seulement un savoir reconnaître ou savoir identifier, mais un savoir décrire, argumenter, raconter, commenter, etc.

3.4 Voilà à grands traits, les attentes à l'égard d'un livre de méthodologie qui ressortent d'un examen des problèmes qui affligent l'enseignement aujourd'hui. C'est sans nul doute par rapport à ces attentes que le livre de Normand Lacharité sera reçu et évalué par un grand nombre de lecteurs.

IV

4. Le livre, Introduction à la métbodologie de la pensée écrite, de Normand Lacharité " propose des exercices conçus pour développer les habiletés de lecture et d'écriture» (p. ix). En particulier, pour la lecture, ces exercices

- «développeront seulement des habiletés qui concernent la reconnaissance de l'organisation des textes sur les plans respectivement rhétorique, thématique et logique» (p. ix);

et pour l'écriture, ils

- «développeront seulement des habiletés qui concernent la mise en place d'une recherche orientée vers la production d'un texte» (p. ix).

Ces dernières habiletés «sont celles qui requièrent les opérations suivantes : la problématisation des thèmes, la recherche de documentation, la détermination de stratégies intellectuelles pertinentes et l'organisation des divers éléments du texte» (p. ix, x). 


\subsection{L'ouvrage est divisé en trois parties :}

4.1.1 La première partie, Les exercices : instructions et éclaircissements, " décrit les tâches que l'étudiant est invité à accomplir en manière d'exercice et fournit, précise $M$. Lacharité, de brèves explications souvent de type lexical, nécessaires à la compréhension des instructions » $(\mathrm{p} . \mathrm{x})$. La description des exercices est faite sous «forme d'instructions» (p. $\mathbf{x})$.

4.1.2 La deuxième partie, Démonstrations et corrigés, présente les textes utilisés pour réaliser certains exercices. (Il s'agit de textes de J.L. Austin, «Performatif-constatif», in La philosophie analytique, Paris, Éditions de Minuit, 1962, p. 271-281) et Bertrand Russell, Problèmes de la philosophie, Chapitres 1 et 2, New York, Oxford University Press, 1943). Elle présente aussi « les résultats de l'effectuation des exercices qui sont du type analyse de texte" ( $\mathrm{p} . \mathrm{x}$ ). Cette partie comprend, comme l'indique son titre, des « démonstrations » et des « corrigés ». Cette distinction tient, écrit M. Lacharité, à ceci : que « les "démonstrations" sont des analyses faites devant les étudiants, sur un texte donné, avant que ceux-ci ne fassent le même exercice sur un autre texte », alors que les «corrigés », conformément à l'usage traditionnel de ce terme, "effectuent l'exercice même demandé aux étudiants » (p. x).

4.1.3 La troisième partie, Essais théoriques et didactiques, est composée de deux textes dont l'un «fournit davantage de détails techniques et de considérations théoriques» par rapport aux exercices d'analyse et l'autre " tente d'élucider le plus concrètement possible la notion de méthode » (p. x) par rapport à la question de la production d'un texte écrit.

4.2 Le matériel pédagogique proprement dit se trouve dans les deux premières parties. Les textes qui constituent « la troisième partie peuvent servir en partie de matériel pédagogique (...) et en partie de considérations didactiques » ( $\mathrm{p} . \mathrm{x}, \mathrm{xi})$.

4.3 La quasi totalité du matériel présentée dans cet ouvrage a été produite, dit M. Lacharité, «à l'occasion de l'enseignement (...) dispensé aux sessions d'automne 82 et 83 " (p. xi) dans le cadre du cours Initiation au travail philosophique (Université du Québec à Montréal). Les exercices ont été réécrits en 1983 et en 1984.

4.4 Si cette réécriture conserve les traces des circonstances premières de production - le vocabulaire, les tournures, les exemples, les textes analysés sont tous "philosophiques" - il ne sera pas exact de supposer ni que le destinataire demeure l'apprenti philosophe, ni que ce matériel réécrit constitue une initiation au travail philosophique (en ce sens qu'il présente les procédures et prorocoles définitoires du travail philosophique et en assure chez l'étudiant une capacité et un niveau de manipulation jugés convenables par la communauté philosophique). Ce livre se situe en deçà des divisions en champs de savoir et propose d'introduire à la méthodologie de la pensée écrite, méthodologie dont l'intérêt et l'utilité ne seront pas limités aux seuls philosophes. Autrement dit, cette méthodologie sera commune à toute pensée écrite, elle sera donc, en quelque sorte indépendante de ses applications ou actualisations dans tel ou 
tel champ particulier. Maîtrisée dans un champ, cette méthodologie sera transférable à un autre.

4.5 La méthodologie que propose $\mathrm{M}$. Lacharité porte sur la réception et la production de la pensée écrite. Elle répond aux questions :

- comment faire pour lire un texte?

- comment faire pour écrire un texte?

Cependant, il ne s'agit pas de toutes les formes de la pensée écrite. Cette méthodologie ne s'applique pas non plus à tous les textes, elle s'applique en tout premier lieu aux textes théoriques, c'est-à-dire, à ces textes qui «ont pour but d'établir, par une mise en contexte et une argumentation, des assertions qui disent, sur le mode indicatif et par des concepts généraux, ce qui est le cas à propos d'une chose ou d'un état de choses pris comme objets de recherche " (p. 207). Le texte ou discours théorique se distingue du texte ou discours « poétique, romanesque, prophétique, pamphlétaire, encyclopédique, électoral, religieux, journalistique, etc. » (p. 207).

La méthodologie que propose $\mathrm{M}$. Lacharité ne portera donc pas sur tous les types de lecture (lecture informative, lecture anecdotique, lecture de détente, lecture critique, lecture productrice, etc.) ni sur tous les types d'écriture (écriture poétique, romanesque, descriptive, injonctive, régulative, expressive, etc.).

Il s'agira presqu'exclusivement de lecture et d'écriture «théorique » ou encore «argumentative».

4.6 Pour lire efficacement un texte "théorique » ou "argumentatif », selon M. Lacharité, il faut être en mesure de reconnaître son organisation «sur les plans respectivement rhétorique, thématique et logique» (p. ix). Les exercices sont conçus spécialement pour développer ces capacités ou ces habiletés.

4.7 Pour écrire efficacement un texte « théorique » ou « argumentatif », il faut être en mesure de problématiser des thèmes, de chercher la documentation, de déterminer les stratégies intellectuelles pertinentes et d'organiser les divers éléments du texte (p. $\mathbf{x})$. Les exercices sont conçus spécialement pour développer ces capacités ou ces habiletés.

4.8 Ainsi, comme on le voit, n'importe quel texte, pourvu qu'il ait quelque chose à dire et qu'il appuie de quelque manière ce qu'il dit peut faire l'objet de la méthode Lacharité. Cette méthode s'appliquera tout autant aux textes «spécialisés » qu'à ceux qui font partie d'un régime très ordinaire de lecture. Elle en rendra la lecture plus efficace. Ce livre n'intéressera donc pas seulement les universitaires et les étudiants à l'université, mais aussi tous les lecteurs ordinaires qui souhaitent rendre leur lecture plus efficace.

4.9 Les capacités que la méthode Lacharité compte développer sont définitoires des activités de lecture et d'écriture. Ne pas savoir reconnaître l'organisation d'un texte théorique, c'est ne pas savoir lire. De même, 
l'écriture théorique se définit comme un ensemble de capacités - problématisation des thèmes, recherche de la documentation, détermination de stratégies intellectuelles, organisation des éléments du texte - qui permettent la production d'un texte organisé sur les plans rhétorique, thématique et logique. Ne pas savoir faire ces choses, c'est ne pas savoir écrire.

$\mathrm{V}$

5. Cette détermination des activités de la lecture et de l'écriture a l'immense mérite de restituer aux textes « rhétoriques » et «argumentatifs » une dimension que les théories dominantes du texte oblitéraient. En effet, en appuyant sur le fait de «lectures plurielles», ces théories ont pris le texte, tout texte, pour un objet aléatoire et la lecture pour une activité essentiellement productrice. Lire n'était pas reconnaître, mais surtout agir, c'est-à-dire s'approprier les éléments d'un texte à la lumière de ce que le texte fait au lecteur, par rapport aux intérêts, préoccupations, désirs, histoire personnelle, etc. de celui-ci. Lire, c'était déterminer en un sens ce qui, comme une matière première, était essentiellement indéterminé.

5.1 L'étudiant qui prend le livre de $\mathbf{M}$. Lacharité apprendra que le texte théorique n'est pas un objet arbitraire ou fortuit qui s'approprie selon les goûts, les intérêts ou les dispositions des urilisateurs; il apprendra que le texte théorique, toujours placé dans une situation de communication, est plutôt un objet construit, organisé selon des règles supposées communes dans un but de dire quelque chose et d'appuyer de quelque façon ce qu'il dit.

5.2 Il apprendra que l'appropriation adéquate, la lecture ou encore la compréhension d'un tel texte, consiste à reconnaître ce que le texte fait (organisation rhétorique), ce dont parle le texte (organisation thématique) et ce que le texte en dit (organisation logique). En particulier, pour l'organisation rhétorique (ce qui est fait) il apprendra que:

- il y a une multitude de procédés d'exposition:

- introduire, illustrer, opposer, rappeler, par exemple;

et que :

- le choix des procédés d'exposition se fait par rapport:

- au thème traité ;

- à la position du locuteur à l'égard du thème ;

- à la position du locuteur à l'égard de l'interlocuteur (le changement à produire);

Pour l'organisation thématique (ce dont le texte parle), il apprendra que :

- il y a une hiérarchie de thèmes définie ou déterminée par le fait qu'un thème ou un groupe de thèmes est «dominant " et que d'autres sont «emboîtés »;

et que :

- il y a une différence entre ce dont parle un texte et ce qu'il en dit, ou ce qu'il soutient à l'égard de ce dont il parle. 
Enfin, pour l'organisation logique (ce qui est dit) il apprendra que :

- certaines " propositions », ou parties du texte, appuient, justifient, prouvent, établissent d'autres propositions ou parties du texte ; les premières s'appellent " raisons ", les secondes s'appellent " conclusions";

et que :

- les liens qui relient les prémisses à une conclusion ont une «force» allant du «nécessaire » au «probable».

5.3 À partir du moment où le texte est placé dans une situation communicationnelle, c'est-à-dire, pris comme moyen et forme d'interaction sociale, un certain nombre d'évidences portant sur les «conditions de possibilité » de cette interaction s'impose. La plus importante est sans doute celle-ci: l'interaction langagière ne peut avoir lieu que sur le fond d'un ensemble de suppositions relatives aux savoirs et aux savoir-faire nécessairement partagées et reconnues comme telles par les interlocuteurs. Ces savoirs et savoir-faire concernent la langue (grammaire et lexique), les choses qu'on fait et qu'on peut faire dans la langue (les actes discursifs) et les situations de communication (champ discursif, locuteur, interlocuteur (réel ou possible)). Autrement dit, dans toute situation communicationnelle, le locuteur suppose que son interlocuteur (réel ou seulement possible) est en mesure de reconnaître :

- ce qui est fait (asserter, donner une raison, avertir, etc.);

- ce dont il est question (principalement, secondairement);

- ce qui en est dit (principalement, secondairement).

L'interlocuteur, de son côté, suppose que le locuteur est en mesure d'employer la langue de telle manière que l'interlocuteur puisse reconnaître, sans se méprendre:

- ce qui est fait (asserter, donner une raison, avertir, etc.);

- ce dont il est question (principalement, secondairement);

- ce qui en est dit (principalement, secondairement).

5.4 Dans les descriptions qu'il fait de l'organisation rhétorique, thématique et logique du texte, $M$. Lacharité rappelle ces évidences. Il est vrai que l'étudiant pourra beaucoup apprendre dans ce rappel - par exemple, qu'une appropriation impressionniste d'un texte ne suffit pas pour le comprendre, qu'un texte n'est pas une suite quelconque de mots et de phrases, mais un ensemble organisé dans le but de faire quelque chose, qu'il y a des «procédés d'exposition ", qu'on peut les identifier, qu'il y a des relations rhétoriques et des relations logiques, que tout ce qui est dit dans un texte n'est pas dit sur le même plan, etc.

5.5 Mais il s'agit là d'évidences, d'informations anciennes et incontestables.

5.6 La réponse que $M$. Lacharité propose à la question : «Comment faire pour lire et pour écrire?" constitue par contre une information nouvelle. 
C'est cette réponse qu'il faur accepter ou refuser, nuancer, restreindre ou amplifier. Pour ce faire, il faut ici poser une autre question : « Est-ce que les instruments, les démarches, les procédés que $\mathrm{M}$. Lacharité propose suffisent pour assurer, autant que faire se peut, l'acquisition des savoir-faire?» Ou encore, «Est-ce que l'effectuation des exercices proposés par M. Lacharité débouche sur une compétence véritable?»

5.7 C'est par rapport à la capacité de la méthode Lacharité de former les compétences ou de contribuer à le faire que les enseignants et les étudiants choisiront d'employer ce livre dans un cours comme manuel, ou comme référence. Autrement dit, c'est l'efficacité de ce livre comme instrument de formation qui déterminera sa réception par les enseignants et les étudiants. Cette méthode sera utile, importante, intéressante, indispensable dans la mesure où elle permettra aux enseignants de mieux accomplir leurs tâches de formation et où elle rendra l'apprentissage, pour l'étudiant, plus aisé, plus commode, plus sûr.

5.7.1 Tourefois, le jugement que les enseignants ou les étudiants feront de ce livre devra tenir compte des deux déterminations du terme " méthode » que $\mathrm{M}$. Lacharité rend explicites dans son « Essai sur les méthodes de lecture et d'écriture de la pensée et particulièrement des contenus philosophiques » (pp. 205-235). Selon M. Lacharité, il faut distinguer « deux contextes d'emploi du terme "méthode" » (p. 205). D'une part, il y a le contexte descriptif où le terme " méthode » désigne " une suite d'opérations reconnues comme ayant été accomplies de fait " (p. 205), et d'autre part, un « contexte praxéologique ou contexte de "design" » dans lequel "on appelle "méthode" une suite d'opérations présentées comme devant être accomplies en vue d'une fin quelconque » (p. 205). Dans le premier contexte, «le terme "méthode" a une acception factuelle»; dans le deuxième, il "possède (alors) une acception prescriptive » (p. 205). De plus, M. Lacharité affirme que c'est de l'acception prescriptive «qu'on a besoin, en contexte d'apprentissage, pour répondre à une question du praticien qui, voulant apprendre, demande: "Comment ferai-je?" Le contexte pédagogique exige que l'on conçoive le design d'une action particulière, à savoir l'apprentissage » (p. 206).

Le jugement qui sera fait de ce livre doit porter alors, d'une part, sur la méthode que propose $\mathrm{M}$. Lacharité, sur le « design » des « opérations présentées comme devant être accomplies » en vue de lire et d'écrire, sur ce que Lacharité dit qu'il faut dire pour lire et pour écrire un texte théorique, et, d'autre part, sur la « méthode » que $\mathrm{M}$. Lacharité suit, les opérations qu'il accomplit pour atteindre son but, « fournir à l'étudiant des éléments de méthode pour lire et écrire efficacement et au professeur des instruments pour développer méthodiquement ces habiletés » (p. ix).

5.8 Il convient de rappeler, pour mémoire, le détail de la méthode Lacharité. Elle consiste essentiellement à :

- "uriliser le système de numération à deux points décimaux pour identifier les suites matérielles du texte» (p. 3) et, précisément, pour identifier les parties er sous-parties du texte; 
- établir l'organisation du texte sur les plans:

- rhétorique (les procédés d'exposition - les coupures et les articulations, ce que le texte fait);

- thématique (ce dont il est question principalement et secondairement) ;

- logique (ce qui est dit ou soutenu, les raisons ou les raisonnements dans le texte) ;

- faire des représentations arborescentes de ces plans d'organisation.

VI

6. Il est certes possible de trouver le « code » pour découper les textes système de numérotation à deux points décimaux - d'une grande complication. Voire d'une application si compliquée qu'il devient l'objet unique d'attention faisant ainsi oublier le texte. Il se peut que l'utilité de ce «code», d'une identification ou découpage si minutieux, si subtil, ne soit pas évidente pour tous. Mais cela n'empêchera pas les réalisations des opérations de reconnaissance de l'organisation du texte sur les plans rhétorique, thématique et logique de coïncider avec l'acte de lire (ou d'écrire) un texte théorique, ou encore, de constituer l'acte de lire un texte théorique. C'est ainsi que l'application de cette partie de la méthode Lacharité, ou encore la capacité d'appliquer cette méthode à un texte sera en quelque sorte une garantie d'une compétence minimale à l'égard des textes. Autrement dit, ce que la méthode Lacharité présente comme «suite d'opérations devant être accomplies » en vue de lire (ou d'écrire) un texte théorique, sera en tout point conforme à cette activité/ pratique sociale.

6.1 L'intérêt majeur, sinon la nouveauté de la méthode Lacharité se trouvera surtout dans la mise en évidence systématique des actes discursifs, dans le fait qu'elle part de l'évidence que le texte fait des choses comme asserter, prouver, illustrer, et que lire c'est reconnaître ce que le texte fait.

La valeur de la méthode Lacharité ne fait pas de doute; cette méthode est en vérité celle qu'emploie tout lecteur un tant soit peu attentif et soucieux de bien " prendre » le texte tel qu'il est donné, de suivre le parcours discursif qui y a été aménagé. C'est cette méthode qu'un locuteur emploiera pour aménager un parcours discursif, pour s'assurer que son « texte » soit « recevable » par son interlocuteur. C'est ainsi que cette méthode définit un modèle de compétence discursive.

La méthode Lacharité n'est rien d'autre que l'expression systématique des règles que nous suivons, des opérations que nous accomplissons dans une communication normale, que nous transgressons dans une communication paradoxale, que nous négligeons ou bafouons dans une communication perturbée.

6.2 En somme, l'intérêt, la valeur et l'utilité de cette méthode se trouvent dans la mise en évidence du fait que le langage est un instrument, un moyen, pour nous, de faire des choses comme asserter, prouver, contester, commenter, etc. et que la réussite de ces actes discursifs n'est possible que si 
l'interlocuteur reconnaît que telle suite de mots ou de phrases est, dans cette situation interlocutive, une assertion, une preuve, un commentaire, etc.

6.3 L'étudiant qui apprend à employer cette méthode sera en mesure de suivre un parcours discursif, il pourra y voir un véritable "prétexte» producteur d'autres textes. L'enseignant qui fait connaître cette méthode rendra à ses étudiants un service d'une valeur inestimable - comme les services rendus par d'autres instances pédagogiques - celles qui montrent à marcher, à courir, à différencier le rouge du bleu, le plein du vide...

\section{VII}

7. Toutefois la méthode que M. Lacharité emploie pour faire apprendre cette méthode, pour « fournir à l'étudiant des éléments de méthode pour lire et pour écrire efficacement, et au professeur des instruments pour développer méthodiquement ces habiletés" ( $\mathrm{p}$. ix) comporte un certain nombre de difficultés. En effer, la méthode que $M$. Lacharité suit, sa pédagogie si on veut, ne semble pas convenir, en tous les cas, à l'atteinte de ses buts - elle ne peut satisfaire les attentes des enseignants - une imprégnation active, une assimilation des procédures, débouchant sur une compétence véritable (et non sur la simple capacité de reproduire des échafaudages ou des constructions). Ces difficultés relèvent non pas de la méthode Lacharité, de sa mise en œuvre ou de sa complexité, mais, pour l'essentiel, d'une conception des moyens d'acquisition qui ne semble pas suffisamment étayée, qui ne tient pas suffisamment compte ni du niveau de l'étudiant, ni de ses capacités. Ou encore, M. Lacharité semble se faire une idée de l'apprentissage d'une pratique, de la formation des savoir-faire, qui ne tient pas suffisamment compte du rapport entre une connaissance théorique des règles et une mise en pratique de celles-ci. Il ne tient pas suffisamment compte du fait que ce genre d'apprentissage se fait en faisant des choses et en faisant faire des choses.

7.1 En effet, M. Lacharité travaille avec l'idée que, dans un contexte pédagogique, pour répondre à la question «comment ferai-je? », il suffit de faire trois choses :

1. Présenter une suite d'opérations comme devant être accomplies en vue d'une fin (p. 205);

1.1 Présenter ces opérations sous une forme prescriptive ou injonctive : «faites ceci»; « chercher cela»; « on subdivise »; « on numérote», etc.

2. Montrer comment « ces opérations » permettent d'atteindre le but envisagé, ou encore montrer que cette méthode s'applique, de fait.

3. Fournir certains éclaircissements et précisions d'ordre lexical.

$7.2 \mathrm{Ce}$ que M. Lacharité fait, c'est montrer comment sa métbode s'applique, mais il ne montre pas comment l'appliquer. La différence est semblable à celle entre montrer qu'une scie peut faire des coupes composées et montrer comment faire, avec cette scie, des coupes composées. La question «comment ferai-je?» doit être prise dans ce deuxième sens. Prise dans ce 
deuxième sens, la méthode sera différente. Parce que $M$. Lacharité ne choisit pas ce deuxième sens, l'étudiant et l'enseignant rencontreront des difficultés dans l'emploi de sa méthode et pourront même la trouver inutile.

7.3 Certes il y a une coïncidence entre le fait de savoir que l'acte de lire consiste à reconnaître l'organisation du texte sur les plans rhétorique, thématique et logique et l'acte même de lire. Toutefois, il y a aussi une « distance » entre savoir ce qu'il faut faire pour lire ou pour écrire et l'acte de lire ou d'écrire - distance indiquée par cette expression quasi-aphoristique de Robert Blanché, «La connaissance des règles suffit à l'arbitre mais non aux joueurs ».

7.4 Il est possible d'illustrer le problème de la méthode qu'emploie M. Lacharité pour faire l'acquisition de la métbode Lacharité comme suit :

Le problème pour beaucoup d'étudiants n'est pas de savoir ce qu'est un thème, le problème est de pouvoir identifier le thème de tel ou tel texte.

Plus précisément, certains étudiants ne peuvent pas identifier le thème dans des phrases simples, comme celles-ci par exemple. *

1. À quand la rentrée?

1.1 Les réponses à la question: «De quoi parle cette phrase?» varient :

- la rentrée

- la date, l'heure de la rentrée

- la date, l'heure

- l'école, etc.

2. «Il n'a aucune faiblesse, il ne fume pas, ne boit ni café ni alcool, il ne lit pas de romans... 》 (D. Buzzati, Les nuits difficiles)

2.1 Les réponses à la question: "De quoi parle cette phrase?" varient :

- il ;

- un type;

- les faiblesses du type ;

- l'absence des faiblesses du type;

3. «C'est dans le secteur universitaire que la loi 15 , qui permet à toute personne de conserver son emploi après 65 ans, risque d'avoir les effets les plus négatifs ». (Lysiane Gagnon, La Presse, 22 novembre 1983)

3.1 Les réponses à la question « De qui parle cette phrase ? " varient:

- la loi 15;

- l'opinion de Lysiane Gagnon ;

- le secteur universitaire ;

- le lieu où la loi 15 risque d'avoir les effets les plus négatifs;

- la retraite non-obligatoire à 65 ans, etc.

\footnotetext{
* Questions posées dans le cours PHI 1601 Raison logique et pensée critique donné à I'Université d'Ottawa aux étudiants de la Faculté des Arts (cours obligatoire) depuis 1983.
} 
Ces exemples montrent assez clairement qu'il ne suffit pas de dire :

- «Identifier le thème "; "Le thème est ce dont parle le texte »;

- "Le thème n'est pas ce que le texte fait ou me fait»;

- «Il n'est pas non plus ce que le texte dit».

Il faut faire plus.

7.5 L'acquisition de la méthode Lacharité et son assimilation pratique se feraient plus aisément et plus efficacement si M. Lacharité complétait les exercices par une série de travaux pratiques allant du simple au plus complexe.

7.6 Plus précisément, pour assurer cet apprentissage et le rendre plus aisé, il faudrait décomposer les opérations de reconnaissance des plans d'organisation. C'est-à-dire commencer avec des plus petites unités, d'une part, et, d'autre part, avec des textes plus familiers. Il faut aussi montrer à l'étudiant une très grande variété de textes et de situations interlocutives pour qu'il puisse se rendre compte des différentes manières de donner un thème, de tenir un propos, de produire des effets discursifs. Il faut lui proposer un très grand nombre de tâches pour assurer l'acquisition de ces habitudes.

7.7 Il faut, de plus, lui donner les moyens de « contrôler » sa lecture par la mise en évidence des contraintes linguistiques et contextuelles qui font que tel groupe de mots est un thème, tel autre ne l'est pas, ou encore celles qui font que tel groupe de mots est une question, une assertion, un appui, etc. Pour la même raison, il faut montrer comment les suites - les répliques, commentaires, actions, etc. - sont des indices de ce qui est en position de thème, de propos tenus, d'actes langagiers posés, etc.

VIII

8. Quel que soit le rapport entre le savoir et le savoir-faire, l'acquisition d'un savoir-faire ne semble pas être le résultat des mêmes démarches ou procédures suivies dans l'acquisition d'un savoir. S'il suffit de dire - décrire, définir, expliquer, prescrire, illustrer, etc. - pour que l'acquisition d'un savoir soit faite (et on sait que ce savoir est acquis lorsqu'il est reproduit), pour nécessaire qu'il soit, le dire ne suffit pas à l'acquisition d'un savoir-faire. Celle-ci demande en plus du dire, un faire et un faire-faire.

8.1 Le problème pour le lecteur ou l'utilisateur éventuel de ce livre est de savoir comment le prendre. Car, comme on le voit, il ne saura pas, à partir de ce qui est fait dans ce livre, s'il doit le prendre comme une présentation de la méthode Lacharité, une sorte de mode d'emploi ou suite d'instructions pour démonter et monter un texte théorique, comportant des illustrations, ou bien comme un ensemble de stratégies et dispositifs mis en place pour favoriser, chez l'étudiant, une maîtrise pratique de la méthode, c'est-à-dire pour favoriser la formation d'une compétence en lecture et en écriture. 
8.2 L'enseignant qui prend ce livre non comme un ensemble d'informations ou d'injonctions, mais comme un moyen d'apprendre et de faire apprendre ces savoir-faire constitutifs de la lecture et de l'écriture devra le compléter et le réaménager pour qu'il serve de cette façon. Deux exemples suffiront à faire voir la nécessité et la nature de ces compléments et réaménagements.

8.3 D'une part, il devra fournir des illustrations des « opérations devant être accomplies » et de la plupart des concepts employés pour présenter ou pour définir ces opérations. Il y sera obligé parce que $\mathrm{M}$. Lacharité en fournit peu et que, dans un contexte pédagogique, il ne suffirait pas de dire qu'une relation logique, par exemple, est :

- "la relation entre une thèse et un argument à l'appui de la thèse (un argument s'appelle aussi "justification", une "raison"); noter qu'un exemple peut servir d'argument, même si ce type d'argument est le plus souvent défectueux du strict point de vue logique » (p. 190).

L'enseignant, pour s'assurer que l'étudiant voit cette relation, dira, au moins: "Dans la phrase: Jean-Marie est venu me voir parce qu'il a des ennuis, il a des ennuis est une raison, un motif, une justification pour la venue de Jean-Marie; parce que signale cette relation».

Ou encore, il ne suffirait pas de seulement dire que «les principales relations rhétoriques sont :

$-\mathrm{x}$ illustre $\mathrm{y}$.

- $\mathrm{x}$ est relié à y par association d'idées.

- $x$ est une explication de $y,(\ldots)$.

- $x$ est un commentaire de y (...).

- $x$ est une digression par rapport à y (...).

— $\mathrm{x}$ est une variation sur y (...) (p. 192-193)

Il faut donner des exemples. Car il est plus que probable que l'étudiant ne comprendra pas ce qu'est « une relation rhétorique » parce qu'il ne sait pas en quoi consistent les opérations d'illustrer, d'associer des idées, d'expliquer, de commenter, etc. Tout au moins il ne le sait pas avant que l'enseignant ne le lui rappelle avec des exemples.

8.4 D'autre part, M. Lacharité rompt avec l'ordre habituel de présentation suivi dans un livre ayant des visées pédagogiques. Comme on le sait, l'auteur d'un tel livre présente, le plus souvent, en tout premier lieu, le champ dans lequel l'étudiant va travailler, les problèmes et les difficultés qu'il peut rencontrer, les concepts avec lesquels il va travailler; l'auteur prend soin de les définir, lorsqu'il s'agit de concepts techniques ou peu familiers, il en donne des exemples ou illustrations, etc. Ensuite, il invite l'étudiant à effectuer certaines tâches qui favoriseront l'acquisition ou l'amélioration (théorique et/ou pratique) des habiletés dont il est question. Dans tous les cas, il présente d'abord des informations, ensuite il demande à l'étudiant de 
les mettre en œuvre. Ce n'est pas l'ordre suivi par M. Lacharité. Il propose d'abord des exercices, ensuite des explications.

8.5 Il n'est pas évident que cette nouvelle forme de présentation permette de mieux atteindre les objectifs pédagogiques annoncés par M. Lacharité. Tout au contraire, elle semble en être un empêchement. Pour atteindre ces objectifs pédagogiques l'enseignant est obligé de réorganiser le livre en présentant ou en faisant lire la troisième partie avant de faire faire des exercices. M. Lacharité lui-même le fait par des renvois et des incises.

Ainsi, dès la première page des «Exercices: Instructions et éclaircissements » (p. 3), M. Lacharité renvoie le lecteur à la troisième partie du texte (sans toutefois lui donner les numéros de page). C'est là où il trouve non pas des «explications additionnelles» mais les informations nécessaires pour comprendre les instructions et pour les suivre. C'est aux pages 163, 164, 165 et 166 que le lecteur trouve les informations qu'il lui faut pour « utiliser le système de numérotation à deux points décimaux pour identifier les suites matérielles du texte... » (p. 3). Le lecteur ne pourra ni identifier ni décrire les coupures et articulations rhétoriques d'un texte, comme le lui demande M. Lacharité, sans prendre connaissance au préalable de la deuxième section "La reconnaissance de l'organisation rhétorique d'un texte » qui se trouve dans la troisième partie de cette méthodologie (pages 166 à 179).

La suite de ce premier exercice, de même que les trois autres exercices présentent pour le lecteur le même problème : il ne sait pas quoi faire sans se rapporter à la troisième partie. C'est pourquoi cette «nouvelle forme d'organisation» semble être un empêchement à l'atteinte des objectifs pédagogiques que $M$. Lacharité s'est donnés.

8.6 Enfin, certaines «informations », bien qu'elles soient données le plus souvent entre parenthèses ou dans la marge, semblent rompre avec les procédés d'exposition qui dominent l'ensemble du texte. Par exemple, les remarques à la page 8 - «(La notion de discours principal est expliquée en classe (...)) », et "Le déblayage (...) constitue un brouillon du travail (...) et n'est pas remis au professeur...»-, ressemblent à des notes ou aidemémoire que l'enseignant consigne dans les marges de ses propres notes de cours. Ces informations données de cette façon posent la question du destinataire de ce livre en ce sens qu'on ne sait pas exactement s'il est adressé aux enseignants à titre de guide ou aux étudiants à titre d'exercices à accomplir.

8.7 Cette question se trouve exacerbée dès que le lecteur se rend compte que les exercices pour développer des habiletés de lecture et d'écriture peuvent être pris comme des instructions qui règlent une série de tâches ou d'opérations que l'utilisateur de ce manuel pourra effectuer sur n'importe quel texte (théorique - argumentatif). Car M. Lacharité fournit « un mode d'emploi " qui s'applique à tous les textes théoriques et argumentatifs; l'utilisateur du livre (professeur ou étudiant) est pour ainsi dire tenu de fournir lui-même les textes sur lesquels il appliquera cette méthode (les 
textes de Russell et de Austin qui font l'objet d'analyse chez M. Lacharité, «sont, dit-il, trop longs pour la plupart des contextes pédagogiques», page 88). M. Lacharité ne fournit pas, à vrai dire, un ensemble d'exercices propres à assurer l'acquisition de cette mérhode.

8.8 La force et la valeur "pédagogique » de ce livre seraient grandement augmentées si M. Lacharité l'enrichissait de « décompositions » et d'exemples. L'enseignant fera cet enrichissement de toute manière. Mais si, par ce fait, il se sent plus libre dans son choix de contenus, il peut tout aussi bien penser que, faute d'instruments assez précis, son travail pédagogique risque d'être plus difficile.

8.9 Il suffirait de quelques réaménagements mineurs, de quelques exemples des relations centrales, pour que ce livre, même sans " exercices ", contribue davantage à résoudre le problème d'incompétence - celle qui rend la tâche de l'enseignant difficile et la jouissance d'un texte impossible.

Département de philosophie

Université d'Ottawa 